\section{Monoartritis del codo e infarto óseo como presentación inicial de enfermedad linfoproliferativa}

\author{
PAULINA DÍAZ ASENCIO ${ }^{1, a}$, OSCAR CONTRERAS OLEA ${ }^{2}$, \\ JOSEFINA DURÁN SANTA CRUZ ${ }^{1}$
}

\section{Elbow monoarthritis and bone infarction as a form of presentation of B cell lymphoma. Case report}

We report a 68-year-old woman presenting with pain and swelling in her left elbow. An elbow magnetic resonance with gadolinium evidenced bone marrow infiltration and a bone infarct. Given these findings, a body CT scan was performed which showed multiple mesenteric adenopathies and a large retroperitoneal mass. A lymph node biopsy confirmed a B cell lymphoma. Monoarthritis with no systemic manifestations represents a highly uncommon form of presentation of lymphoma. Moreover it usually affects inferior limbs, particularly in the presence of bone infarction.

(Rev Med Chile 2017; 145: 795-798)

Key words: Arthritis; Bone Marrow Diseases; Elbow; Lymphoma; Lymphoproliferative Disorders.

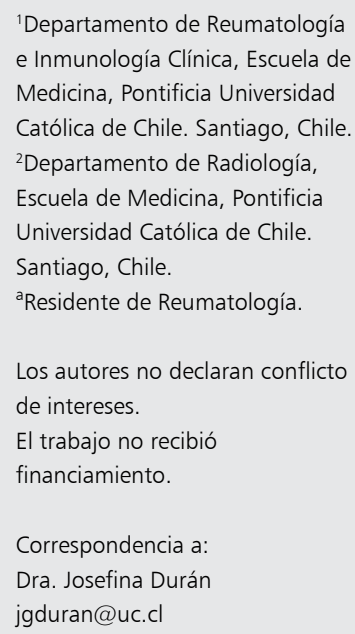

$\mathrm{P}$ resentamos un caso clínico de síndrome linfoproliferativo que debutó como una monoartritis de codo, donde la resonancia magnética (RM) fue clave para llegar al diagnóstico. En esta oportunidad, se revela la importancia de la evaluación de la médula ósea en la RM que se realiza como parte del estudio de una artritis.

\section{Caso clínico}

Comunicamos el caso de una paciente de 68 años de sexo femenino, previamente sana, que consultó por un cuadro de 5 días de evolución de intenso dolor en el codo izquierdo. Al exámen físico, presentaba notoria inflamación y eritema en dicha región, con dolor a la palpación e impotencia funcional. Además a la inspección, se constató lesión erosiva de la piel por una quemadura causada por vapor de agua. No tenía historia de fiebre ni calofríos.
Dentro de los exámenes iniciales de laboratorio, destacaba una anemia leve con $\mathrm{Hb}$ de $11,2 \mathrm{~g} / \mathrm{dL}$, sin leucocitosis, con glóbulos blancos $8.600 \mathrm{x} \mathrm{mm}^{3}$, con fórmula diferencial normal, $201.000 \mathrm{x} \mathrm{mm}^{3}$ plaquetas y VHS de $39 \mathrm{~mm} / \mathrm{h}$. El frotis sanguíneo era normal. Se solicitó factor reumatoideo y anti péptidos cítricos citrulinados (antiCCP), los cuales fueron negativos. Una radiografía del codo evidenció esclerosis ósea y osteofitos. Por último, la ecografía de codo mostró derrame articular, sin sinovitis. Se realizó una punción articular, cuyo estudio de líquido sinovial fue de tipo inflamatorio, con $4.350 \mathrm{~GB} \mathrm{x} \mathrm{mm}^{3}$ y $81 \%$ de polimorfonucleares $(\mathrm{PMN})$. No se evidenciaron cristales. El cultivo del líquido fue negativo.

Con estos hallazgos se diagnosticó una celulitis de dicha región y una artrosis de codo. Se inició tratamiento con cefadroxilo por 10 días y antiinflamatorios no esteroidales, con disminución importante de la inflamación y el dolor.

A los treinta días, la paciente consultó nue- 


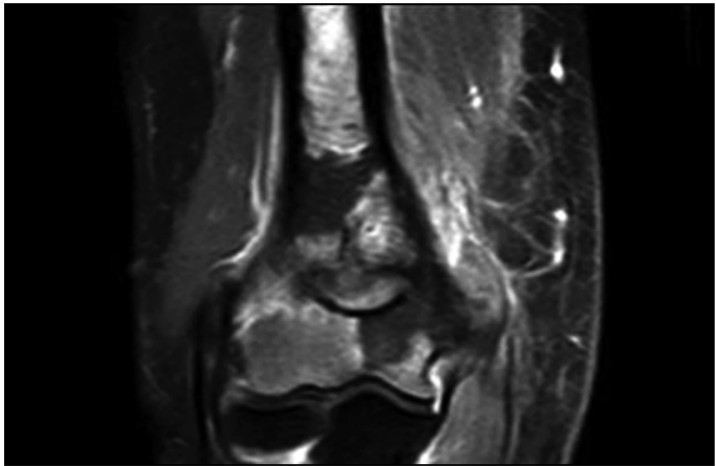

Figura 1. Imagen de resonancia magnética de codo en el plano coronal, en secuencia T1 con saturación grasa y gadolinio. Se observa sustitución difusa de la médula ósea del húmero distal, con áreas geográficas que no presentan reforzamiento con el uso de contraste, manifestación de focos de necrosis medular.

vamente con inflamación y severo dolor en la misma región.

$\mathrm{Al}$ examen físico, se evidenció artritis de codo, destacando un dolor exquisito a la presión digital en la región epitroclear.

Se realizó una resonancia magnética con gadolinio, que evidenció infiltración de la médula ósea, de aspecto hipercelular, sugerente de enfermedad linfoproliferativa y áreas con ausencia de impregnación posterior a la administración de gadolinio, sugerentes de infarto en la cara medial de la epífisis distal del húmero (Figura 1). A su vez, se evidenció abundante líquido sinovial y engrosamiento de la membrana sinovial (Figura 2).

Un nuevo hemograma mostró en el frotis sanguíneo linfocitos inmaduros con núcleos segmentados y regular cantidad de citoplasma.

Se realizó una tomografía axial computada (TAC), que reveló múltiples adenopatías mesentéricas con un diámetro sobre $9,5 \mathrm{~cm}$ y una gran masa retroperitoneal. Se realizó una biopsia de ganglio linfático, la cual confirmó un linfoma de células B.

\section{Discusión}

Ya en el año 1983, se describe la asociación entre manifestaciones reumatológicas y enfermedades hematológicas ${ }^{1}$, sin embargo, a la fecha actual, la evidencia en la literatura más bien hace referencia a reportes de casos clínicos.

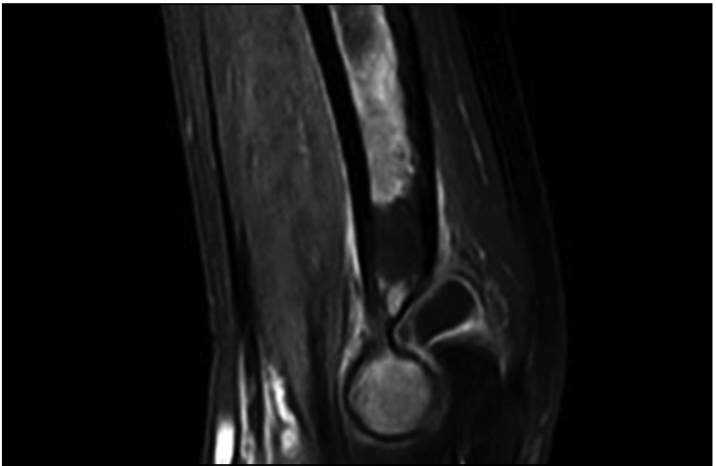

Figura 2. Imagen de resonancia magnética de codo en el plano sagital, en secuencia T1 con saturación grasa y gadolinio. Se observa moderado derrame articular en el codo, con engrosamiento y reforzamiento de la sinovial articular, lo que es compatible con sinovitis.

Se ha descrito la asociación de manifestaciones reumatológicas en una serie de enfermedades hematológicas, dentro de ellas, la anemia de células falciformes, talasemia, hemocromatosis, leucemia aguda, mieloma múltiple, hemofilia, linfoma, existiendo en algunas de estas enfermedades, asociaciones bien conocidas, en otras más bien su ocurrencia anecdótica ${ }^{2}$.

Respecto al linfoma, se ha reportado que entre 5-25\% de los pacientes con linfoma no Hodgkin presentan manifestaciones musculoesqueléticas ${ }^{2,3}$. Entre ellas, la más frecuentemente reportada es el dolor óseo, y con menor frecuencia, la presencia de monoartritis, o poliartritis ${ }^{2}$. Típicamente, el compromiso óseo es secundario, siendo la infiltración articular rara y más bien una reacción sinovial por compromiso del tejido óseo subyacente ${ }^{4}$.

Respecto a las articulaciones comprometidas, de los pocos casos descritos en la literatura, la articulación afectada con mayor frecuencia es la rodilla. En una revisión de los casos publicados hasta el año 2013, se cuentan 18 casos con presencia de mono o poliartritis asociada a linfoma no Hodgkin, siendo en 13 de ellos la presencia de artritis considerado como manifestación precoz de enfermedad subyacente. Sólo en 7 casos se reportó compromiso monoarticular, sin otras manifestaciones de linfoma no Hodgkin. La articulación afectada con mayor frecuencia fue la rodilla (3 casos), esternoclavicular $(2$ casos $)$ y codo $(2 \text { casos })^{4-7}$. 
En estos casos, la biopsia sinovial y ósea es un elemento clave en el diagnóstico, sin embargo, las actuales técnicas imagenológicas, pueden orientarnos al diagnóstico, como en el caso presentado. Las alteraciones de la médula ósea en la resonancia nuclear magnética pueden ser altamente sugerentes de infiltración medular, como ocurrió en este caso, donde solamente en base a este hallazgo se realizó un estudio dirigido con TAC y posterior biopsia de ganglio.

En el caso expuesto destaca, además, la presencia concomitante de infarto óseo. El proceso de necrosis ósea y de tejido medular, habiéndose excluido infección, se denomina infarto óseo, particularmente cuando se ubica en la diáfisis y metáfisis oseas. El mismo proceso también es conocido como necrosis avascular o aséptica cuando se ubica en la epífisis ${ }^{8-10}$. A pesar de existir esta diferenciación en la terminología, muchas veces en la literatura, se describe este proceso de forma indistinta ${ }^{11}$.

Hoy en día se han identificado poblaciones de riesgo para presentar infarto óseo y necrosis ósea avascular, estando documentados en pacientes con anemia de células falciformes, talasemia, enfermedad de Gaucher, pacientes diabéticos, enfermedades mieloproliferativas, trasplante renal y uso de terapia crónica esteroidal ${ }^{9-12}$.

El infarto óseo puede presentarse en forma crónica, siendo clínicamente asintomático u oligosintomático, y visualizándose en forma incidental por exámenes imagenológicos. También puede presentarse en forma aguda, con dolor intenso, localizado en una o más áreas del esqueleto. Esta presentación puede acompañarse de sensibilidad a la palpación, inflamación y eritema sobre el sitio del infarto, como en el caso presentado, siendo también común la presencia de fiebre y leucocitosis ${ }^{13}$. Cuando el infarto óseo incluye la epífisis, puede existir la presencia de líquido articular ${ }^{8}$.

La presencia de infarto óseo se ha reportado principalmente en extremidades inferiores, en $77 \%$ de los casos $^{14}$. Las radiografías convencionales son poco sensibles para detectar infarto óseo en forma aguda, pudiendo visualizarse, más bien infartos óseos crónicos, mostrando las lesiones antiguas, márgenes escleróticos y calcificados delimitando el segmento necrótico.

La apariencia del infarto óseo está bien descrita como la presencia de un delgado borde de baja señal en secuencias potenciadas en $\mathrm{T} 1$ y una señal variable en el centro. Adicionalmente, en las señales potenciadas en T2 el signo característico de la "doble línea" sugiere fuertemente la posibilidad de infarto óseo medular".

El signo de la doble línea ocurre en la interfaz entre el tejido viable y no viable. Consiste en un borde externo de baja señal, que corresponde a hueso esclerótico con un borde interno de señal de alta intensidad, correspondiente a tejido de granulación vascularizado o metaplasia condroide.

La RM es esencial en las fases iniciales del infarto, permitiendo caracterizar las lesiones y proceder a diagnóstico temprano ${ }^{15}$. Si bien el hallazgo imagenológico es de gran utilidad para orientar el estudio, es necesario la confirmación mediante biopsia para descartar malignidad u osteomielitis ${ }^{9}$.

\section{Referencias}

1. Isenberg DA, Shoenfeld Y. The rheumatologic complications of hematologic disorders. Semin Arthritis Rheum 1983; 12: 348-58.

2. Menon S, Isenberg DA. Rheumatological manifestations of haematological diseases. Ann Rheum Dis 1995; 54: 787-95.

3. Visser J, Busch VJ, de Kievit-van der Heijden IM, ten Ham AM. Non-Hodgkin's Lymphoma of the synovium discovered in total knee arthroplasty: a case report. BMC Research Notes 2012; 5: 449.

4. Neri R, Giorgetti M, Zampa V. Case Report.Elbow monoarthritis revealing B cell non-Hodgkin's lymphoma Clin Rheumatol 2013; 32 Suppl 1: S111-4.

5. Jawa A, Lieberman AE, Alexieva CC, Jupiter JB. Primary intra-articular non-Hodgkin's lymphoma of the elbow. A case report. J. Bone Joint Surg Am 2006; 88: 2730-4.

6. Rice DM, Semble E, Ahl ET, Bohrer SP, Rothberger H. Primary lymphoma of bone presenting as monoarthritis. J Rheumatol 1984; 11: 851-4.

7. Haasa KK, Durk H, Baumbach A, Saal J, Wehrmann M Non-Hodgkin's lymphoma presenting as knee monoarthritis with a popliteal cyst. J Rheumatol 1990; 17: 1252-4.

8. Almeida A, Roberts I. Bone involvement in sickle cell disease. Br J Haematol 2005; 129: 482-90.

9. Hara H, Akisue T, Fujimoto T, Kishimoto K, Imabori $\mathrm{M}$, Kishimoto $\mathrm{S}$, et al. Magnetic resonance imaging of medullary bone infarction in the early stage. Clinical 
Imaging 2008; 32: 147-51.

10. Lafforge P, Trijau S. Bone infarcts: Unsuspected gray areas? Joint Bone Spine 2016; 83: 495-9.

11. Saini A. Saifuddin A. MRI of osteonecrosis. Clin Radiol 2004; 59: 1079-93.

12. Umans H, Haramati N, Flusser G. the diagnostic role of gadolinium enhanced MRI in distinguishing between acute medullary bone infarct and osteomyelitis. Mag Reson Imaging 2000; 18: 255-62.
13. Kim SK, Miller JH. Natural history and distribution of bone and bone marrow infarction in sickle hemoglobinopathies. J Nucl Med 2002; 43: 896-900.

14. Ibn Yacoub Y, Rostom S, Amine B, Hajjaj-Hassouni N. Uncommon case of tibial bone infarction associated with juvenile idiopathic arthritis. Clin Rheumatol 2010; 29 (4): 427-9.

15. Almirall $\mathrm{M}$, Perich $\mathrm{A}$, Carbonell J. Infartos medulares óseos. Rev Esp Reumatol 2002; 29: 444-8. 